\title{
ASPECTOS CRÍTICOS DO USO DE CAQDAS NA PESQUISA QUALITATIVA: UMA COMPARAÇÃO EMPÍRICA DAS FERRAMENTAS DIGITAIS ALCESTE E IRAMUTEQ
}

\author{
Angela Canuto', Beatriz Bragal ${ }^{1}$, Lucas Monteiro' ${ }^{1}$ e Rodrigo de Melo' \\ 1'Faculdade de Medicina da Universidade Federal de Alagoas, Brasil. Angela_canuto@uol.com.br; beatriz.braga@famed.ufal.br; \\ lucas.monteiro@famed.ufal.br; ropparanhos@gmail.com.
}

\begin{abstract}
Resumo. Introdução: Atualmente, na metodologia da pesquisa qualitativa, o uso de Softwares de Análise de Dados Qualitativos Assistidos por Computador (CAQDAS) é vital para analisar dados, seja através do ALCESTE, IRAMUTEQ ou outras ferramentas. Contudo, assim como toda ferramenta, as CAQDAS apresentam pontos positivos e negativos. Assim, torna-se importante analisar o uso destes softwares na pesquisa qualitativa. Objetivos: Analisar funções e aplicação de CAQDAS como componentes da metodologia em pesquisas qualitativas. Métodos: Comparação entre as funções do ALCESTE e IRAMUTEQ e análise crítica do uso desses CAQDAS em artigos qualitativo e quali-quantitativo produzidos pelos autores. Resultados: Ambos os artigos tiveram resultados convincentes decorrentes do uso de CAQDAS. Ademais, o IRAMUTEQ apresenta mais funções, quando comparado ao ALCESTE. Discussão: O uso de CAQDAS na pesquisa qualitativa pode ser de suma importância, principalmente em trabalhos que lidam com grandes quantidades de textos. Além disso, a escolha do software a ser utilizado depende do tipo de resultado desejado. Conclusão: Percebe-se a utilidade do uso de CAQDAS em pesquisas qualitativas, garantindo dinamismo no processamento de dados textuais. O uso correto destas ferramentas é fundamental para a análise dos dados coletados e posterior reprodução científica de pesquisas na mesma linha de interesse.
\end{abstract}

Palavras-chave: Pesquisa Qualitativa; Análise de Dados; Software de Análise Qualitativa de Dados - QDA

\section{CRITIC ASPECTS OF CAQDAS USAGE IN QUALITATIVE RESEARCH: AN EMPIRIC COMPARISON OF ALCEST AND IRAMUTEQ AS DIGITAL TOOLS}

Abstract. Introduction: Recently, usage of Computer-assisted qualitative data analysis software (CAQDAS) became an important data-analysis strategy in qualitative research methodology, whether by ALCESTE, IRAMUTEQ or other analytic tool usage. However, as with any other device, positive and negative aspects will always be present. Knowing this, it is important to analyze the usage of those softwares in qualitative research. Objectives: Evaluate function and application of CAQDAS as part of methods in qualitative research. Methods: Function comparison between ALCEST and IRAMUTEQ, alongside critic analysis of CAQDAS in qualitative and quali-quantitative research papers produced by the present authors. Results: Both analysed papers had crucial usage of the chosen CAQDAS. Furthermore, IRAMUTEQ presents itself with a greater number of inner tools, when compared to ALCESTE. Discussion: CAQDAS usage can become crucial, specially in research requiring bigger amounts of text. Also, there is not a "superior software", in qualitative analysis, as the software of choice depends on the expected results. Conclusion: The utility and importance of digital analytic tools is seen during article development and researches that demand skill and dynamism in textual data management. Its correct usage also provides credence in further methods reproduction.

Keywords: Qualitative Research; Data Analysis; Qualitative Data Analysis Software - QDA 


\section{INTRODUÇÃO}

A pesquisa qualitativa é o tipo de abordagem metodológica que busca compreender o ponto de vista dos participantes, bem como seu contexto social, sobre determinado tema, sendo essa perspectiva individual então interpretada pelas experiências do pesquisador (Camargo, 2013). Inicialmente, o ramo qualitativo era visto como uma subdivisão da pesquisa quantitativa, entretanto, ela é considerada, atualmente, como sua própria vertente metodológica. Dessa forma, a gradativa evolução do método qualitativo atua como uma espécie de desmistificação da soberania estatística da análise quantitativa, que era tida, até meados da década de 1980, como a única forma confiável de coletar dados (Justo, 2014).

A análise de conteúdo de Bardin, popularizada a partir de 1977, considerada o método clássico de análise qualitativa, é uma técnica de descrição sistemática de termos e classes de equivalência. Ela é trabalhada em duas etapas: a primeira inclui a definição das classes de equivalência e identificação de suas ocorrências; já a segunda envolve a contagem de cada uma dessas classes e construção de uma tabela que sistematize a análise. Ainda que a técnica fosse primorosa e fácil de ser utilizada, apresentava certas limitações, como falta de rigor metodológico e dificuldade na análise de bancos de dados mais extensos (Justo, 2014).

Dessa forma, o surgimento de softwares voltados à essa vertente científica, no fim do século $X X$, permitiu, além da solução das limitações apresentadas na análise de Bardin, que houvesse maior confiabilidade e maiores possibilidades na análise de dados. Pois, a possibilidade de se usar um programa de computador para contar e agrupar dados, no lugar do pesquisador, permitiu que este pudesse investir mais tempo na interpretação dos dados gerados, expandindo a porção analítica da pesquisa, além de dar mais confiabilidade científica a seu trabalho com a automatização da coleta de dados, antes manual. Por conta disso, o método qualitativo digital vem se difundindo de maneira cada vez mais ampla e célere (Souza, 2018).

Esses programas são os Softwares de Análise de Dados Qualitativos Assistidos por Computador (CAQDAS) e permitem que os autores organizem, compilem, codifiquem e gerenciem vários tipos de dados, como áudios, imagens, vídeos e textos, de modo que sua ação sobre os dados coletados expanda-se e flexibilize-se. Tais programas computacionais possuem diversas vantagens sobre a pesquisa analógica, como a maior facilidade na busca de termos específicos, o aumento da velocidade de separação dos dados e codificação e 
até a uniformidade caligráfica. Seja através do ALCESTE (Analyse Lexicale par Context d'un Ensemble de Segments de Texte), do IRAMUTEQ (Interface de R pour les Analyses Multidimensionnelles de Textes et de Questionnaires) ou de outro Software de ação similar, a análise digital de textos, nesse caso, torna-se mais coerente, concisa e compreensível para o público-alvo da pesquisa (Souza, 2018). ALCESTE e IRAMUTEQ foram escolhidos porque, além de serem softwares de destaque em relação aos outros CAQDAS, compartilham a função de "Classificação Hierárquica Descendente" ou CHD, como explicitado por Camargo (2013).

Dessa forma, o presente artigo pretende analisar os pontos positivos e negativos de duas CAQDAS amplamente utilizadas pela comunidade científica (ALCESTE e IRAMUTEQ), através de análise das ferramentas que cada software apresenta, bem como pela experiência dos autores com o uso desses programas como parte da metodologia de dois artigos que escreveram. Deve-se ressaltar, além disso, que não há software objetivamente superior ao outro, dado que cada um apresenta um modo de operação próprio. Dessa forma, a escolha do programa deverá ser determinada pela finalidade, necessidades e objetivos de estudo em questão e que, nesse estudo, a comparação será feita tomando como base as experiências dos presentes pesquisadores na escrita dos dois artigos.

\section{OBJETIVOS}

\subsection{Objetivo principal}

Analisar os pontos positivos e negativos de duas CAQDAS muito usados pela comunidade científica (ALCESTE e IRAMUTEQ), a partir da análise das ferramentas internas de cada software e das experiências dos autores com o uso dessas ferramentas como parte da metodologia de dois artigos que escreveram.

\subsection{Objetivos específicos}

- Avaliar a logística de uso dos softwares ALCESTE e IRAMUTEQ;

- Comparar as funções e limitações dos softwares ALCESTE e IRAMUTEQ; 
- Identificar a viabilidade do uso do ALCESTE e do IRAMUTEQ como CAQDAS em pesquisas com teor qualitativo parcial ou integral;

- Evidenciar a experiência prática do uso do ALCESTE e do IRAMUTEQ como CAQDAS.

\section{METODOLOGIA}

A metodologia do presente artigo embasou-se na comparação entre as funções dos softwares ALCESTE (versão 4.0) e IRAMUTEQ (Versão 0.7 alfa 2), ambos usados no sistema operacional Windows 10, a partir da leitura integral de seus manuais, disponibilizados nos sites de seus fornecedores, e da experiência dos autores com suas funções como foco da metodologia de dois artigos escritos pelos próprios pesquisadores.

Neste trabalho, foram estudados dois artigos de pesquisa qualitativa e quali-quantitativa produzidos pelos próprios autores cuja coleta de dados foi realizada, respectivamente, por entrevista semiestruturada e Grupos Focais. O artigo "Aspectos da relação médico-paciente em Hospital Universitário" foi escrito embasado em uma análise qualitativa pura, enquanto o artigo "Análise da empatia dos estudantes de medicina no primeiro ano de curso na Universidade Federal de Alagoas - UFAL" foi criado sob uma ótica quali-quantitativa, com o enfoque em seus aspectos subjetivos.

A realização de transcrições digitais para futuro processamento nos CAQDAS foi feita manualmente usando o software Apache OpenOffice 4.1.7, recomendado no manual de instruções do IRAMUTEQ, para que não haja corrupção de dados durante a análise pelo software.

A análise comparativa entre os dois CAQDAS usados foi feita, finalmente, em duas etapas. A primeira foi realizada através da busca de artigos de relevância nas plataformas virtuais Pubmed e Scielo em português ou inglês com data de publicação entre 2009 e 2019, dandose preferência para artigos com data de publicação a partir de 2015, para seleção e análise literária. Também foram utilizados e referenciados os manuais de instruções do ALCESTE e do ITAMUTEQ, para investigação aprofundada das funções de cada software. Foram utilizados os descritores "Pesquisa Qualitativa", "ALCESTE", "IRAMUTEQ", "Análise Comparativa", "Análise Lexical" e a operação Booleana AND destes para elucidação dos pontos positivos e limitações de cada programa. 
A segunda etapa comparativa consistiu na análise empírica dos dois softwares, a partir de uma comparação dos critérios logísticos de seu uso, da quantidade de funções e limitações. Para isso, foram utilizados os manuais de uso do ALCESTE e IRAMUTEQ, para melhor embasamento da análise empírica, e os artigos produzidos pelos autores, para observar, na prática, as vantagens e desvantagens de cada programa na produção de artigos científicos da vertente qualitativa.

\section{RESULTADOS}

O uso dos CAQDAS foi primordial para o prosseguimento de ambas as pesquisas, dando-se através do uso dos Softwares ALCESTE e IRAMUTEQ, respectivamente. Ambos têm lados positivos e negativos, tornando o uso de cada um deles mais viável a depender da proposta de cada artigo. Nesse sentido, os resultados seguintes contêm uma breve descrição de cada CAQDAS, bem como um tópico dedicado a cada etapa da análise comparativa.

\subsection{ALCESTE}

Desenvolvido por M. Reinert na França no final da década de 1980, o ALCESTE foi apresentado como uma ferramenta inovadora no processo de análise textual. Esse software possibilita uma análise lexográfica e integra uma grande quantidade de métodos estatísticos sofisticados, configurando-se como um instrumento de análises de dados textuais que fornece critérios para que o material em questão seja considerado indicador de um fenômeno de valor científico (Canuto, 2020).

A ideia de funcionamento do ALCESTE também foi sintetizada por Camargo (2005), quando ele comenta sobre a análise de Classificação Hierárquica Descendente (CHD), que permite análise lexical dos textos, além de ofertar classes lexicais, que se caracterizam por vocabulário específico e por segmentos textuais de mesmo vocabulário, o que torna mais fidedigna a interpretação das análises lexicais.

Enquanto técnica, o ALCESTE investiga a distribuição de vocabulários em um texto escrito e em transcrições de texto oral. Como metodologia, integra uma grande quantidade de métodos estatísticos refinados através da segmentação, classificação hierárquica, análise de correspondências, dentre outros recursos, configurando-se em um método de exploração e descrição (Azevedo, 2013). 
O programa, a partir da aplicação da análise lexical, fornece-nos vários dados. Destaque para as Unidades de Contexto Elementar (UCE) que, a partir do pertencimento das palavras de um texto a uma UCE, o programa estabelece as matrizes que serão usadas como base para o trabalho de classificação.

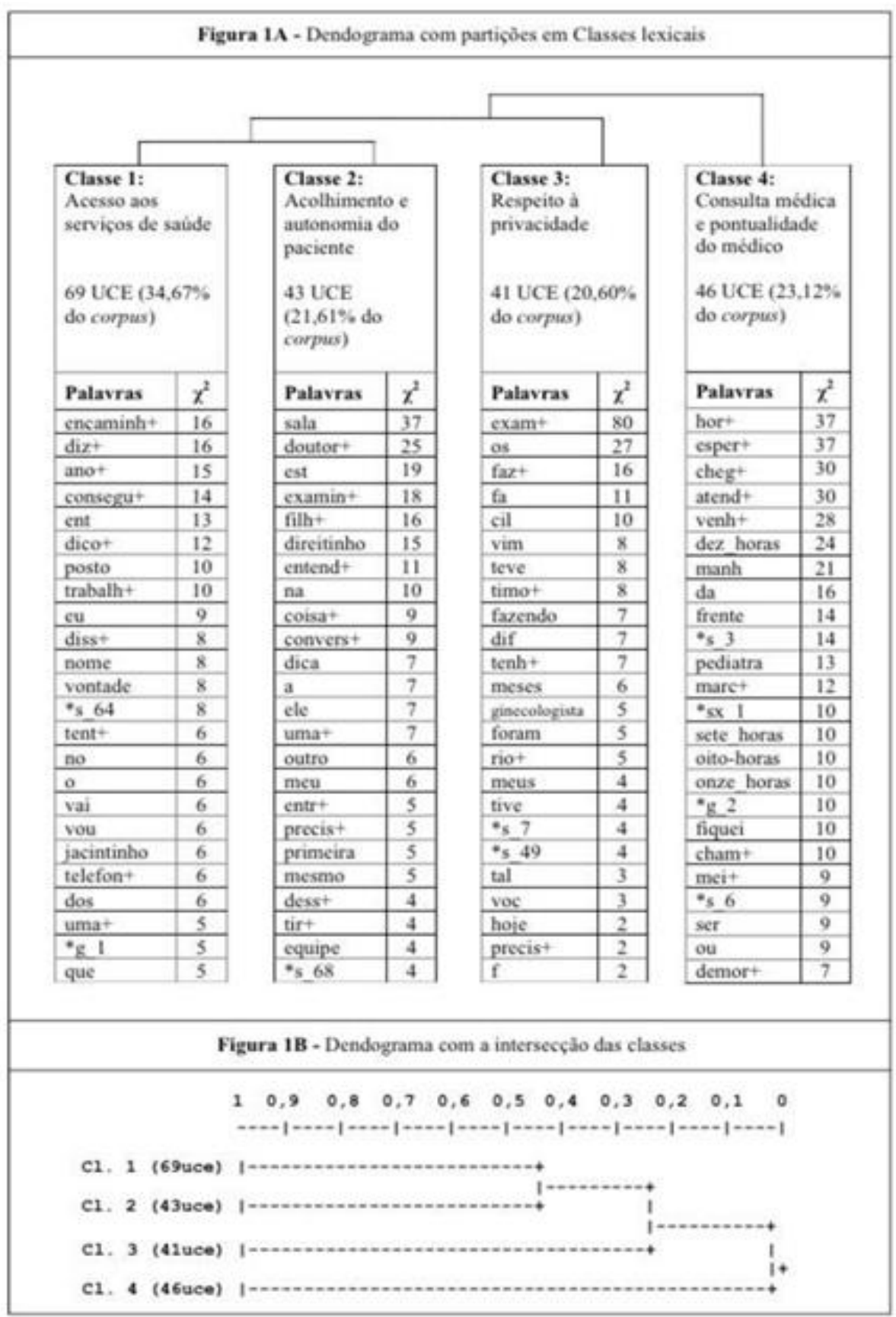

Figura 1. Dendograma com partições em Classes lexicais de artigo sobre a relação médico-paciente em hospital universitário gerado a partir de entrevista semiestruturada, relacionando 4 classes de palavras e suas respectivas UCE. 
No entanto, como mencionado por Pombo-de-Barros (2015), o Alceste pode ser útil nos dando uma visão global sobre uma documentação volumosa, mas não faz Análise Temática de Conteúdo. O software é preterido em pesquisas por ter somente a opção de análise de Classificação Hierárquica Descendente (CHD), enquanto ferramentas mais recentes e atualizadas podem oferecer análises mais complexas e ricas.

\subsection{IRAMUTEQ}

O IRAMUTEQ, criado pelo francês Pierre Ratinaud em 2009, é um Software desenvolvido na linguagem Python e que funciona como uma extensão do Software estatístico R. Atualmente ele apresenta a maioria dos menus em português e tutoriais oficiais dedicados a ensinar sobre sua instalação e uso na mesma língua, o que o torna acessível a pesquisadores de diferentes nacionalidades.

Ele utiliza o mesmo algoritmo do ALCESTE para realizar análises baseadas na Classificação Hierárquica Descendente (CHD), entretanto, também usa esse algoritmo para outros tipos de análises, como descrito por Souza (2018):

\footnotetext{
"Além da CHD, o IRAMUTEQ incluiu outras formas de análises, como as textuais clássicas, de especificidades, similitude e nuvem de palavras. Ambos os softwares são considerados ferramentas de processamento dos dados, e não um método de pesquisa, o que torna seus resultados instrumentos de exploração, busca e associação em material de pesquisa".
}

Dentre as ferramentas do IRAMUTEQ, as de maior destaque são a "Nuvem de Palavra" e a "Análise de Similitude" que, por serem representações visuais dos dados gerados pelo corpus (arquivo criado a partir das transcrições digitais), tornaram a comparação dos resultados obtidos por diferentes grupos melhor e mais intuitiva para sua exposição nos artigos científicos. Isso confere ao IRAMUTEQ maior quantidade de funções para geração de resultados com base em um corpus único, quando comparado às funções do ALCESTE. Essas possibilidades distintas, dada a finalidade e longitudinalidade da pesquisa na qual o IRAMUTEQ foi utilizado, foram o fator primordial para seu uso pelos pesquisadores (Monteiro et al, 2020).

Outro fator positivo para o uso do IRAMUTEQ é o fato de ele ser um software gratuito de acesso aberto (open access, em inglês), o que permite maior disseminação do software 
entre os pesquisadores, uma vez que não existe, nesse caso, o impedimento financeiro para seu uso.

Tratando-se das limitações do IRAMUTEQ, a maior dificuldade enfrentada foi a demanda de tempo necessária para habituação e domínio do software por parte de alguns pesquisadores do grupo, o que é, como exposto por Salvador (2018), o possível fator que dificulte o uso dessa CAQDAS específica para alguns pesquisadores. Essa demanda de tempo necessária pode ser observada durante o processo de instalação do IRAMUTEQ, que requer a instalação do software de análise "R", para que as análises possam ser realizadas, o que pode ser considerado um contratempo para alguns pesquisadores.

A quantidade de funções distintas, ainda que não seja uma limitação em si, pode não ser atrativa para pesquisadores que busquem um programa capaz de realizar uma tarefa mais simples e não disponham de tanto tempo para dominar o IRAMUTEQ, o que motivou o uso do ALCESTE na pesquisa na qual ele foi utilizado.

A nuvem de palavras é um método mais simples de se organizar a análise dos termos, porém é mais graficamente agradável, sendo uma forma útil e concisa de se observar quais as palavras mais frequentes que foram proferidas durante a geração do corpus. Termos mais frequentes são representados mais ao centro e tem um tamanho maior que os menos ditos, que ficam mais à margem da nuvem.

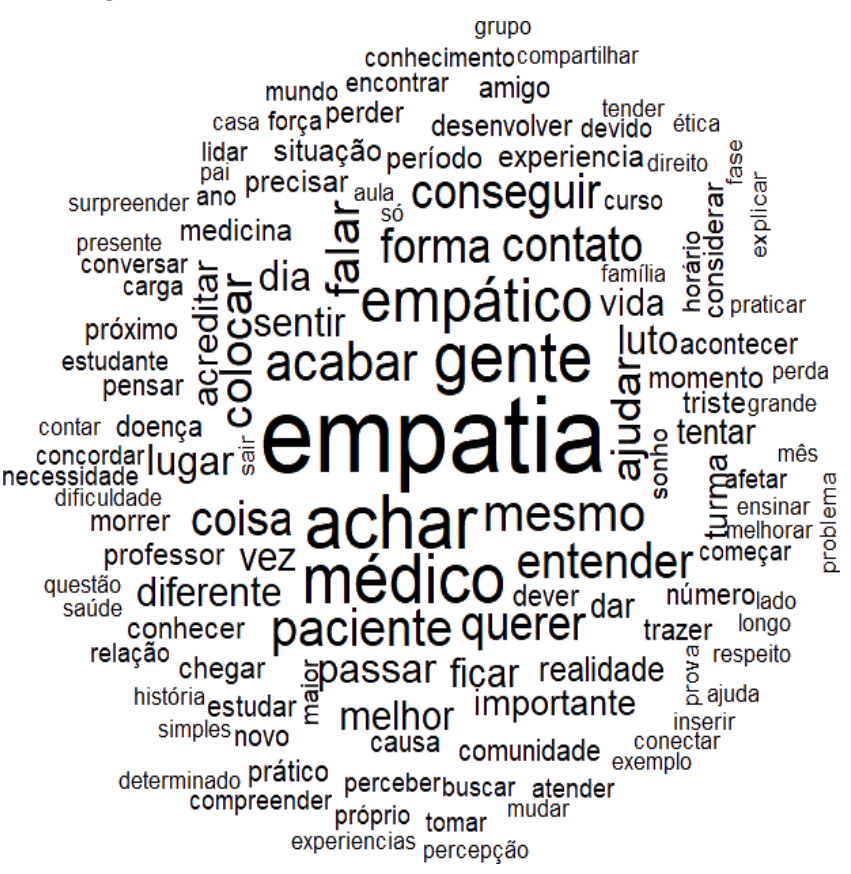

Figura 2. Nuvem de Palavra de um Grupo Focal realizado com alunos do sexo Masculino no $1^{\circ}$ ano da Graduação em Medicina. Mostra a frequência das palavras proferidas durante a realização do Grupo Focal 
Já a Análise de Similitude configura-se como uma forma mais completa dos segmentos, quando comparada ao método anterior, uma vez que permite observar a correlação entre termos diferentes, qual o termo que deu origem à discussão e quais os termos com maior frequência. Além disso, certas opções permitem que formas sejam sobrepostas à "rede" da análise, separando-a em blocos com vértices e arestas que, como descrito no manual de instalação e uso do IRAMUTEQ, indicam a frequência das palavras e força de coocorrência entre elas, respectivamente (Camargo, 2018).

A possibilidade de representação imagética dos dados, substituindo a tabulação de dados presente no ALCESTE, permite maior quantidade de análises distintas com base no mesmo corpus, isto é, uma transcrição pode gerar uma quantidade maior de dados e resultados distintos no IRAMUTEQ, se comparado ao ALCESTE.

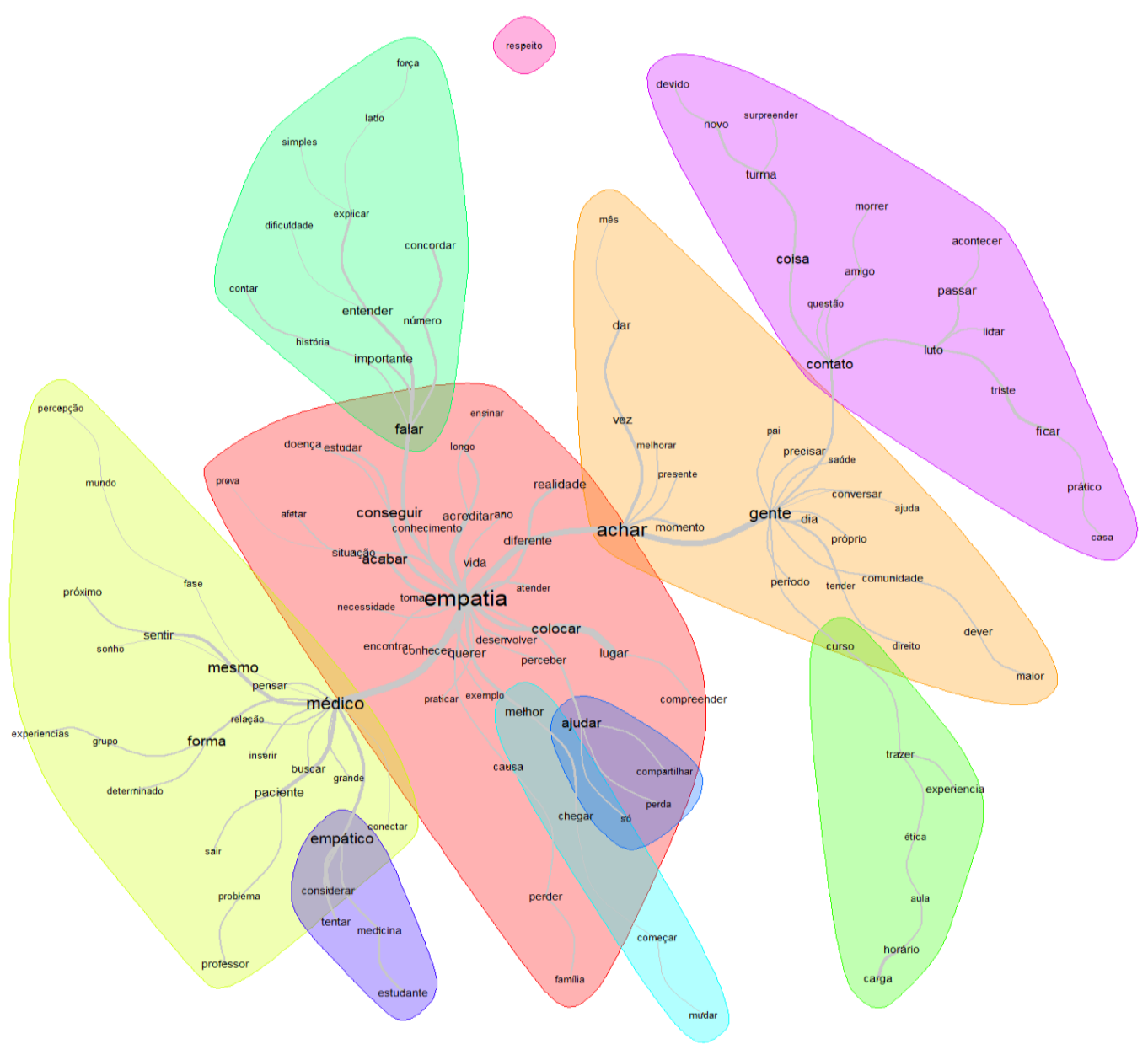

Figura 3. Análise de Similitude de um Grupo Focal realizado com alunos do sexo Masculino no $1^{\circ}$ ano da Graduação em Medicina. Mostra a frequência e correlação entre as palavras proferidas durante a realização do Grupo Focal. 


\section{DISCUSSÃO}

Os pacotes de software para Análise de Dados Qualitativos são ferramentas muito úteis para auxiliar os investigadores que desenvolvem projetos de investigação qualitativa ou mista. Essas ferramentas possuem potencialidades para auxiliar na transcrição, escrita e anotação, codificação e interpretação de texto, abstracção recursiva, análise e pesquisa de conteúdo análise de discurso, mapeamento de dados, metodologia da teoria fundamentada, entre vários outros tipos de análise (Reis, Costa \& Souza, 2016).

Os CAQDAS podem colocar a pesquisa qualitativa em um patamar essencial no meio acadêmico, de forma a ampliar a capacidade de produção, o que auxilia na criação de um arcabouço mais rico para a temática buscada, consequentemente trabalhos mais robustos.

Um fator positivo dos softwares é a segurança de que os resultados sairão conforme o pesquisado, e isto faz a pesquisa qualitativa se destacar frente à pesquisa tradicionalmente restrita ao método quantitativo. Outro ponto importante associado ao uso dos CAQDAS é a possibilidade de trabalhar com uma quantidade elevada de dados, o que também confere maior confiabilidade ao tópico pesquisado. Entretanto, as limitações de cada ferramenta abrem oportunidades para que um tipo de pesquisa não seja objetivamente superior que o outro em todos os aspectos e permite que ambas se aperfeiçoem, para assim impulsionar o lançamento de novas versões desses softwares.

Além de tempo e segurança, o entendimento melhor do custo-benefício de cada método possível de ser utilizado interfere significativamente na escolha ideal de uma metodologia científica em pesquisa qualitativa, sendo a decisão entre os CAQDAS uma etapa primordial da metodologia. Seguindo esse aspecto crítico de escolha, o uso entre ALCESTE e IRAMUTEQ depende do tipo de resultado de se deseja obter.

Nesse sentido, o uso do ALCESTE, dado os seus benefícios e limitações, se enquadra melhor em uma pesquisa que precise ser completada rapidamente e a qual apenas a análise de Classificação Hierárquica Descendente (CHD) seja feito, uma vez que, se o projeto não tiver tempo limite para ser completado, o IRAMUTEQ pode ser utilizado por realizar o mesmo tipo de análise do ALCESTE. Já o IRAMUTEQ pode ser melhor aproveitado em pesquisas que planejem fazer mais de um tipo de análise, que pretendam usar representações imagéticas dos dados e que possuam tempo suficiente para canalizar 
no aprendizado de suas ferramentas, do contrário, o software poderá não cumprir com as expectativas do pesquisador.

Destarte, assim que o pesquisador dominar o CAQDAS escolhido, o tempo ganho no processo de coleta e formatação de dados é utilizado em uma interpretação mais coerente do tema, e essa maior segurança em um processo qualitativo não o assemelha a um quantitativo, mas pode trazer uma maior relevância científica, podendo ser reproduzida em outros estudos, o que põe esse tipo de ciência em um fluxo de melhoria contínua.

\section{CONCLUSÕES}

Os CAQDAS são extremamente importantes para o desenvolvimento de pesquisas qualitativas e quanti-qualitativas, pois permite que o pesquisador, uma vez habituado às ferramentas digitais, possa poupar tempo e canalizá-lo na análise e discussão dos resultados gerados virtualmente.

A respeito do ALCESTE e do IRAMUTEQ, dois dos mais usados softwares na análise do discurso, os resultados obtidos com a comparação de suas funções e com as experiências práticas dos autores no uso de ambos foram de suma importância no processamento dos dados e na geração e organização dos resultados. O softwares foram vitais para o prosseguimento de ambas as pesquisas que, sem eles, não poderiam ter sido finalizadas de forma satisfatória.

Um dos pioneiros na criação de Classes Lexicais, O ALCESTE é um programa extremamente útil para uma análise pontual sobre determinado texto, que no caso funciona como dado de entrada, ou seja, dado ainda não processado. Porém, o software possui alguns obstáculos de utilização, como o fato de ser pago, tornando-o menos acessível que o IRAMUTEQ. Sua função é ideal para pesquisadores que pretendem utilizar a CHD como ferramenta na metodologia de seus projetos, uma vez que, por realizar apenas esse tipo de análise, ele é um software mais específico que o IRAMUTEQ, nesse contexto.

Este, por outro lado, é gratuito e, além de disponibilizar uma análise de CHD como o ALCESTE, possui outras funções de análise, como as visuais, que muitas vezes facilitam na geração e análise de resultados. Por conseguinte, o aumento da acessibilidade e da variedade faz do IRAMUTEQ muitas vezes ser o escolhido quando se toma um comparativo entre os dois softwares. O uso de recursos visuais como resultados auxilia em uma visão 
espacial do material ainda não processado, deixa os resultados mais palpáveis e agrega valor a pesquisa qualitativa. Entretanto, a demanda de tempo necessária para aprender a usar o IRAMUTEQ corretamente pode afastá-lo de pesquisadores com menos tempo ou disposição para dominar todas as funções do IRAMUTEQ, o que pode ser visto como uma limitação.

Ademais, a adesão de CAQDAS representa um progresso na pesquisa qualitativa, porém questões como até que ponto a subjetividade do autor deve ser deixada de lado, se métodos mais analógicos, como a análise de conteúdo de Bardin, não fariam os resultados de cada pesquisa mais singulares e de maior qualidade, ou se o caminho da pesquisa qualitativa é se mesclar a outras metodologias, tornando-a mais completa, devem ser avaliadas se podem ser caminhos possíveis de serem trilhados e o interesse por eles de vital relevância para a literatura, o que demanda novos estudos que possam responder a esses questionamentos.

\section{REFERÊNCIAS}

Azevedo, D. M., Costa, R. D. S., \& Miranda, F. D. (2013). Uso do Alceste na análise de dados qualitativos: contribuições na pesquisa em enfermagem. Rev enferm UFPE, 7(1), 5015-5022.

Camargo, B. V. (2005). ALCESTE: um programa informático de análise quantitativa de dados textuais. Perspectivas teórico-metodológicas em representações sociais, 1, 511-539.

Camargo, B. V., \& Justo, A. M. (2013). IRAMUTEQ: um software gratuito para análise de dados textuais. Temas em Psicologia, 21(2), 513-518

Camargo, B. V., \& Justo, A. M. (2018). Tutorial de uso do Software IRAMUTEQ, Recuperado em 25 fevereiro de 2020, de http://www.iramuteq.org

Canuto, A. M. M., Gêda, T. F., Guimarães, J. E., Mendes, C. O., Manna, T. B. F., \& Monteiro, E. M. (2019). Perspectiva dos Discentes de Medicina de uma Universidade Pública sobre Saúde e Qualidade de Vida. Revista Brasileira de Educação Médica, 43(1, Suppl. 1), 228-235.

Canuto, A. M. M., \& Monteiro, L. (2020). ASPECTOS DA RELAÇÃO MÉDICO-PACIENTE EM HOSPITAL UNIVERSITÁRIO. Revista Portal: Saúde e Sociedade, 4(3), 1194-1205.

Justo, A. M. \& Camargo, B. V. (2014). Estudos qualitativos e o uso de softwares para análises lexicais. Em: Novikoff, C.; Santos, S. R. M. \& Mithidieri, O. B., Caderno de artigos: X SIAT \& II Serpro, 37-54.

Loubère, L. \& Ratinaud, P. (2014). Documentation IraMuTeQ 0.6 alpha 3 - version 0.1 [Computer software]. Recuperado em 19 fevereiro de 2014, de http://www.iramuteq.org

Monteiro, L. N., Braga, B. P., Canuto, Â. M. M., Gêda, T. F., de Melo, R. P., dos Santos, N. S. M., \& Santos, E. C. F. (2020). Análise da empatia dos estudantes de medicina no primeiro ano de curso na Universidade Federal de Alagoas-UFAL/Analysis of empathy of medicine students in the first year of course at the Federal University of Alagoas-UFAL. Brazilian Journal of Development, 6(2), 6972-6984. 
Pombo-de-Barros, C. F. (2015). Análise textual com o Programa ALCESTE: uma aplicação em pesquisa de representações sociais no campo da política. Caminhos para análise das políticas de saúde (1a ed), 427432.

Reinert M. (2007). Postures énonciatives et mondes lexicaux stabilisés en analyse statistique de discours. Langage et société; 3-4(121-2), 189-202.

Reinert M. (2000). Alceste (version 4.0 Windows). Toulouse: Image.

Reinert M. (1990). Alceste une méthodologie d'analyse des données textuel les e tune application: Aurelia de Gerard de Nerval. Bull Methodol Sociol. 26(1):24-54.

Reis, L. P., Costa, A. P., \& Souza, F. D. (2016). Análise comparativa de pacotes de software de análise de dados qualitativos. In Conferência Ibérica de Sistemas e Tecnologias de Informação (CISTI) (pp. 15-18).

Salvador, P. C. O., Gomes, A. T. L., Rodrigues, C. C. F. M., Chiavone, F. B. T., Alves, K. Y. A., Bezerril, M. S. \& Santos, V. E. P. (2018). Uso do software iramuteq nas pesquisas brasileiras da área da saúde: uma scoping review. Revista Brasileira em Promoção da Saúde. 18(1): 1-9

Souza, E. D. S., Rodrigues, M. D. S., Rocha, F. D. C., \& Martins, C. R. (2009). Guia de utilização do software Alceste: uma ferramenta de análise lexical aplicada à interpretação de discursos de atores na agricultura. Embrapa Cerrados-Documentos (INFOTECA-E).

Souza, M. A. R., Wall, M. L., Thuler, A. C. M. C., Lowen, I. M. V., \& Peres, A. M. (2018). O uso do software IRAMUTEQ na análise de dados em pesquisas qualitativas. Revista da Escola de Enfermagem da USP, 52(03353). 